\title{
Representación espacial en los niños ciegos: una investigación sobre las principales variables que la determinan y los procedimientos de objetivación más adecuados
}

\author{
ESPERANZA OCHAÍTA \\ JUAN ANTONIO HUERTAS \\ ANGELES ESPINOSA \\ Universidad Autónoma de Madrid
}

Resumen

El objetivo fundamental de este trabajo es el estudio conjunto de los principales factores que determinan el conocimiento y la representación del entorno en los invidentes, haciendo especial énfasis en las relaciones entre desarrollo y aprendizaje. El marco teórico se toma de la psicología ambiental y de la psicología evolutiva del conocimiento espacial. El procedimiento consiste en el aprendizaje de un entorno desconocido, en un máximo de cuatro sesiones. El conocimiento espacial se evalúa mediante tres tipos de técnicas. Los resultados no muestran, en términos generales, grandes diferencias entre ciegos de nacimiento y ciegos tardios, ni tampoco entre el espacio pequeño y el grande. Las diferencias más significativas son las debidas a la edad de los sujetos, y el aprendizaje también se revela como factor importante, aunque en nuestro diseño parezca estar subordinado al desarrollo. La investigación tiene implicaciones claras para la instrucción de la orientación, movilidad y conocimiento del entorno en los invidentes.

Palabras clave: Ciegos, Representación espacial, Desarrollo.

\section{Spatial representation in blind children: an investigation into the main determining factors and the most appropiate externalization techniques}

\section{Abstract}

Results from a research project focussing on factors determining spatial knowledge and representation of physical environment by blind subjects are presented in this paper, and the relationships between learning and development are the factors studied. The study's theoretical framework is taken from environmental psychology and a developmental approacb to spatial knowledge. The procedure consisted in learning an unknown environmet (large/small) in a maximun of four trials. Spatial knowledge was assessed through three techniques. Overall, results do not show great differences in perfomance between congenital and adventitiously blind subjects, or between small and large environments. The most significant differences are due to subjects' age, and to their previous learning experience, though in our design the latter appears to be subordinated to cognitive development. These results bave clear implications for orientation instruction, mobility, and spatial knowledge in visually impaired subjects.

Keywords: Blind children, Spatial representation, Development.

Agradecimientos: Este artículo forma parte de una investigación más amplia financiada por la C.I.C.Y.T. con el $n$. $^{\circ}$ PB 85-0278, titulado «Ontogénesis y microgénesis de la representación del entorno en las personas ciegas. Agradecemos a las directivas de los colegios de la ONCE de Madrid y Barcelona la colaboración prestada para la realización de este trabajo.

Dirección del autor: Universidad Autónoma de Madrid. Facultad de Psicología. Cantoblanco. 28049 Madrid.

Original recibido: Octubre 1990. Revisión recibida: Diciembre 1990. Aceptado: Diciembre 1990 


\section{INTRODUCCION Y OBJETIVOS}

Ha sido mucha la literatura que desde tiempos muy remotos se ha dedicado al problema del conocimiento del espacio en las personas ciegas. Filósofos tales como Molineux, Locke, Berkely y Diderot se interesaron por este tema y actualmente siguen apareciendo en las revistas psicológicas numerosos artículos que estudian distintos aspectos relacionados con la capacidad de los invidentes para representarse el espacio más o.menos inmediato.

A pesar de la relevancia que este tema tiene para la psicología cognitiva teórica y para la práctica educativa de los invidentes, todavía hoy no contamos con resultados concluyentes acerca de las variables que determinan o modulan la capacidad de las personas ciegas para conocer y representarse el entorno: la edad, la experiencia visual previa, la experiencia con el espacio (aprendizaje), así como el tamaño y la complejidad del mismo. Tampoco se sabe con claridad qué relaciones existen entre la movilidad o conducta espacial de un sujeto en un entorno determinado y la representación mental que tiene de dicho entorno, ni cuáles son las técnicas más adecuadas para «exteriorizar» u objetivar las representaciones espaciales de los ciegos.

Una de las variables más importantes que inciden en el tipo y grado de conocimiento y representación espacial de los sujetos es su nivel de desarrollo ontogenético. En el caso de los videntes, existe un buen número de investigaciones sobre el desarrollo de la representación espacial, que suelen tomar como referencia el modelo constructivista de Hart y Moore (Hart y Moore, 1973; Moore, 1973, y Hart, 1979). Por el contrario, no conocemos investigaciones que estudien el conocimiento espacial de los ciegos desde esta perspectiva evolutiva, a pesar de que resultan imprescindibles, tanto para aclarar el problema de la espacialidad en las personas ciegas como para elaborar programas educativos de movilidad y conocimiento espacial.

Por el contrario, sí se ha dedicado mucho espacio en la literatura experimental a estudiar la incidencia que tiene la experiencia visual previa en la capacidad de los ciegos para moverse y conocer el entorno. Lógicamente, el factor de haber tenido experiencia con el entorno a través del sistema sensorial espacial por excelencia - la visión-, puede condicionar el tipo o nivel de representación que un invidente tenga de un espacio. Sin embargo, no podemos decir que la experiencia visual previa facilite de forma clara la movilidad, conocimiento y representación espacial. Como ya se dijo anteriormente (Ochaíta y Huertas, 1988), las investigaciones realizadas al respecto no ofrecen siempre datos favorables para los ciegos tardíos. En términos generales podemos concluir que el haber tenido experiencia visual previa a la ceguera facilita la representación espacial cuando se trata de un espacio complejo, cuya representación se evalúa por medio de respuestas también complejas, de las que luego se infiere el nivel de coordinación o configuración que el sujeto tiene de ese espacio. Sin embargo, no se han encontrado diferencias entre ciegos precoces y tardíos en el conocimiento y representación de espacios no complicados estructuralmente, en los que se mide un conocimiento de tipo secuencial conformado por una ruta (Fletcher, 1981; Hudson, 1984).

La experiencia o el grado de aprendizaje que se tiene de un entorno es, sin duda, un factor que determina el conocimiento y capacidad de repre- 
sentación del mismo. Intuitivamente, todos sabemos que tenemos representaciones más ajustadas de los espacios que conocemos bien. Los datos experimentales obtenidos con videntes ponen de manifiesto que cuando un individuo se enfrenta a un ambiente espacial nuevo, se reproduce, microgenéticamente, la secuencia de etapas descritas por Hart y Moore para el desarrollo ontogenético. Este aprendizaje será más o menos rápido de acuerdo con el nivel de competencia espacial que posea el individuo (Apleyard, 1969, 1970; Beck y Wood, 1976; Moore, 1973). No obstante, las investigaciones realizadas desde una perspectiva evolutiva (Hart, 1979; Martín, 1985; Aragonés, 1988) han demostrado que la experiencia no determina sin más la capacidad de representación espacial, sino que interactúa con el nivel de desarrollo cognitivo del sujeto.

No hemos encontrado, en el caso de los ciegos, ningún trabajo que haya estudiado la capacidad de los niños de diferentes edades para aprender un entorno. En sujetos adultos, los resultados de Hollyfield y Foulke (1983), ponen de manifiesto que las personas ciegas son capaces de conocer y representar un espacio de forma más adecuada a medida que se. suceden las sesioines de aprendizaje del mismo. No obstante todavía no está claro si los invidentes pueden llegar a tener representaciones del espacio de tipo complejo o configuracional o si, como afirma Foulke (1982), sólo adquieren representaciones secuenciales en forma de rutas.

Es importante también tener en cuenta que el nivel de conocimiento y representación de un espacio está determinado por su tamaño y complejidad y que estos factores son de importancia crucial en ausencia de la visión. En general, las investigaciones realizadas en un entorno pequeño como un laboratorio o una habitación, obtienen mejores resultados para los ciegos si las comparamos con aquellas que se llevan a cabo en espacios grandes, como un barrio o una ciudad. Un factor íntimamente ligado al tamaño del espacio es su complejidad. Los trabajos realizados en este sentido han demostrado que el número de elementos que un entorno tiene, así como las relaciones de esos elementos entre sí, determinan su conocimiento por parte de las personas ciegas (Warren, 1987).

Uno de los problemas más importantes que subyacen en los distintos trabajos revisados es el de las relaciones entre conducta espacial real o movilidad, la representación mental que las personas tienen del espacio en que realizan tal actividad y la objetivación o externalización de esa representación. La importacia del tema ha sido señalada por autores tales como Marchesi, 1983; De Vega, 1984; Aragonés, 1988. En el caso de las personas ciegas, las relaciones entre conducta y representación espacial cobran, si cabe, mayor relevancia. En ausencia de la modalidad sensorial visual (la más idónea para obtener la información sobre el espacio no abarcable con las manos), la persona ciega tiene que suplir, en gran medida, los datos perceptivos mediante el recuerdo y el análisis del conocimiento que tiene almacenado de ese entorno. Por último, hemos de señalar que resulta imprescindible estudiar de forma rigurosa cuáles son los métodos más adecuados para objetivar las representaciones espaciales de los ciegos. Como ya hemos dicho anteriormente (Huertas y Ochaíta, 1988), buena parte de la heterogeidad de los resultados experimentales sobre la capacidad de conocimiento espacial de los ciegos se debe a los diferentes métodos empleados por los 
FIGURA 1
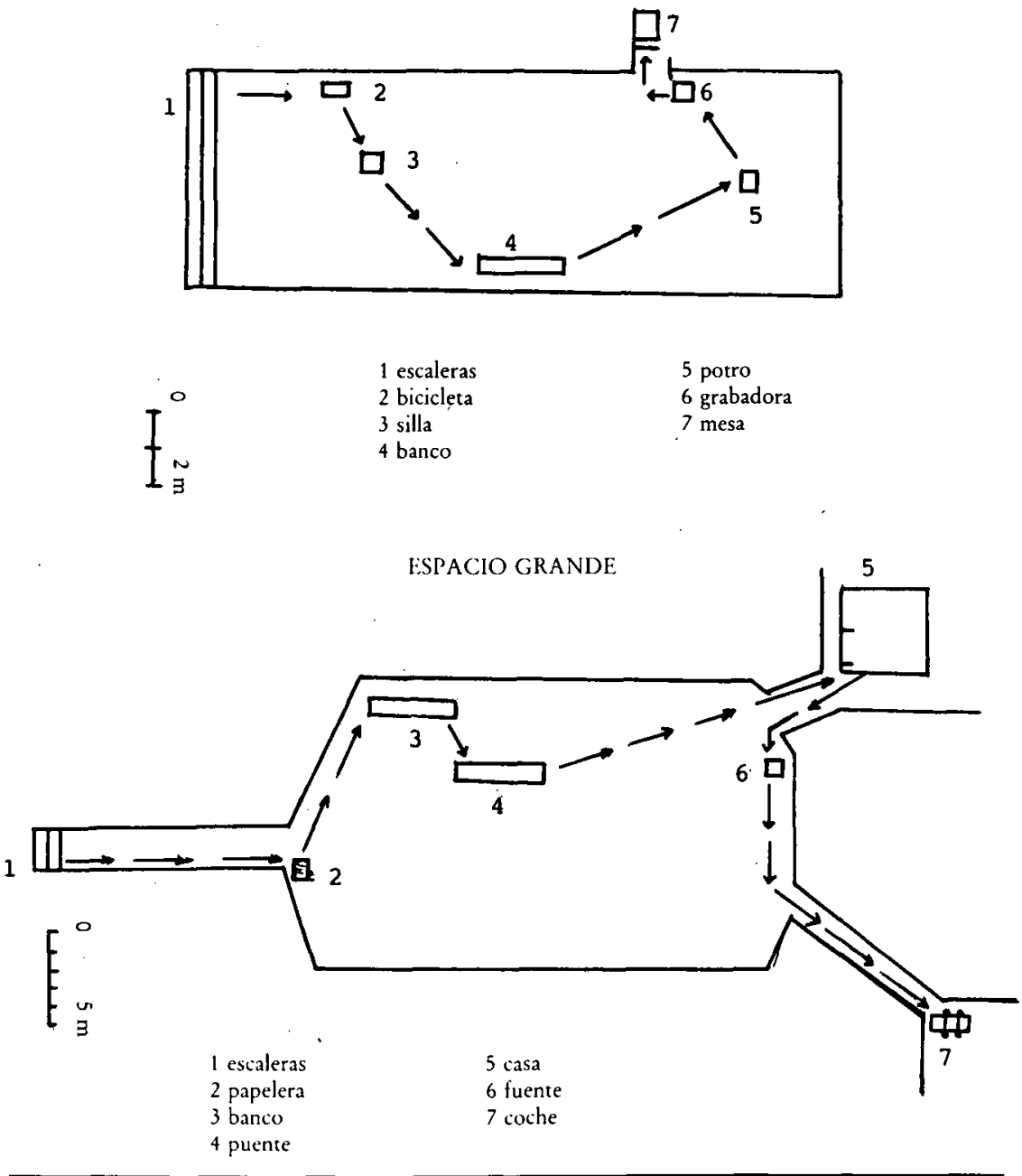

Croquis del recorrido en el espacio pequeño y grande

distintos autores; métodos que no siempre son adecuados a las características de los sujetos estudiados.

En consecuencia con todo lo que se acaba de decir, los objetivos de este trabajo son los siguientes:

En primer lugar, se trata de estudiar conjuntamente las principales variables que parecen determinar la movilidad, el conocimiento y la representación del entorno en las personas ciegas: el nivel de desarrollo (la edad), la cantidad de experiencia con el espacio (aprendizaje), la experiencia visual previa y el tamaño del espacio, haciendo especial énfasis en las relaciones 
entre aprendizaje y desarrollo. En segundo lugar nos proponemos conocer cuáles pueden ser los métodos más adecuados para objetivar o exteriorizar las representaciones espaciales de los ciegos, haciendo especial énfasis en estudiar las relaciones entre movilidad y representación espacial.

Se ha partido de un marco teórico mixto tomado de la psicología de la cognición ambiental (Evans, 1980; Downs y Stea, 1973; Stokols y Altman, 1987, etc.) y de la psicología evolutiva del conocimiento espacial (Piaget e Inhelder, 1947; Piaget, Inhelder y Szeminska, 1948; Moore, 1974; Hart, 1979). De esta última se ha tomado la descripción de las etapas del desarrollo de la representación ambiental. Respecto a las relaciones entre aprendizaje y desarrollo se asume una postura crítica ante el concepto de desarrollo de Piaget y la escuela de Ginebra, tomada en cierta medida, de la psicología de la instrucción y del concepto vigotskiano de «zona de.desarrollo próximo" (Vygotski, 1934).

\section{METODO}

La muestra está formada por dos grupos independientes de sujetos, uno de los cuales realizó las sesiones de aprendizaje en un espacio grande y el otro en uno pequeño (variable «tamaño del espacio»). Cada uno de estos grupos se dividió, a su vez, en otros dos en relación con la variable «experiencia visual»: ciegos tempranos/ciegos tardíos. Se han tomado cuatro niveles de edad para cada uno de los grupos considerados con medias de $9,11,14$ y 17 años respectivamente. El número total de sujetos es de 38 , que conforman casi la totalidad de la población de ciegos con las condiciones experimentales requeridas, que estudian en los colegios de la ONCE en Madrid y Barcelona..

Se utilizaron, como ya se ha dicho, dos espacios de tamaño diferente (grande/pequeño). El espacio grande era una plaza de la ciudad de Madrid, de donde se eligieron siete puntos o «landmarks» que conformaban una ruta con curvas y cambios de dirección. Para controlar el efecto de la complejidad del espacio y variar sólo su tamaño, se imitó la misma disposición espacial con los siete elementos en el soportal que daba acceso al patio de recreo de los colegios, en una superficie de aproximadamente $75 \mathrm{~m}^{2}$ (ver Figura 1).

El procedimiento consistía en que los sujetos aprendiesen el recorrido del espacio que les correspondía en un máximo de cuatro sesiones, llegando a localizar de acuerdo con la ruta diseñada, los siete elementos que la componían. En el primer ensayo era el experimentador el que guiaba al sujeto por el recorrido, mostrándole los diferentes puntos de que constaba la ruta; en los restantes, el experimentador era guiado por el niño. En caso de que el sujeto tuviera problemas con el recorrido, se le proporcionaban una serie de ayudas estandarizadas de diferentes grados según la cantidad de información que proporcionaban. A continuación exponemos algunos ejemplos de estas ayudas ordenadas de menor a mayor cantidad de información que suministran.

- Ayuda 1: Si el sujeto no es capaz de anticipar el punto siguiente al cual debe dirigirse, se le dice cuál es ese punto. 
- Ayuda 2: Si el sujeto se perdía, no encontraba el punto de referencia o no sabía cómo continuar, se volvía con él hasta el mojón anterior y desde alli se indicaba que señalase la dirección en que suponía se encontraba el punto de referencia perdido.

- Ayuda 3: Si volvía de nuevo a no encontrar el elemento correspondiente, se le acompañaba otra vez al mojón anterior y de allí se le llevaba al punto de referencia siguiente.

- Ayuda 4: Si continuaba sin saber cómo llegar a un punto de referencia dos veces más, es decir, si durante tres tramos del recorrido era necesario proporcionarle ayudas 2 y 3 , se volvía con el sujeto hasta el principio del recorrido y se daba una vuelta por toda la ruta de la misma forma que se habia hecho en la primera sesión de trabajo.

- Ayuda 5: Si a pesar de la ayuda 4, el sujeto volvía a manifestar que desconocía cómo llegar a más de uno de los puntos, entonces se le enseñaba tramo por tramo cada recorrido.

Todas las sesiones se registraron mediante una cámara de vídeo y un magnetofón. Se controló, asimismo, el tiempo que duraba cada desplazamiento.

Al final de cada sesión el sujeto tenía que externalizar la representación que había adquirido del recorrido. En las sesiones primera y última realizaba una maqueta y una estimación verbal de distancias de la ruta recorrida. En las sesiones intermedias sólo se utilizaba uno de los dos métodos.

Para comprobar de otra forma el grado de aprendizaje que había alcanzado el sujeto, debía realizar un recorrido distinto a modo de comprobación, cuando terminaba la última sesión. Se trataba de enlazar en una ruta nueva, tres de los puntos del recorrido (en concreto, debía ir desde el elemento 7 al 5 y de éste al 3). Se suponía que si lo lograba plenamente, el sujeto habría alcanzado cierto nivel de coordinación de ese espacio, ya que se mostraba capaz de utilizar una ruta alternativa.

Para estudiar la movilidad o recorrido se han utilizado cuatro procedimientos distintos: a) La observación estructurada de las grabaciones en vídeo de los recorridos hechos por los sujetos, realizada por tres jueces, de acuerdo con cuatro categorías: "tropiezo", "pasarse del punto", "perderse», «desvío» y «marcha normal»; b) La contabilización del número y tipo de ayudas recibidas; c) Duración del recorrido, y d) El grado de éxito alcanzado en el movimiento durante la última sesión, de acuerdo con los siguientes criterios ordinales de éxito.

- Nivel 9: El sujeto no necesita ninguna ayuda durante su último recorrido, incluido el recorrido de comprobación. Tampoco ha cometido incorrecciones importantes en su desplazamiento, la categoría de perderse y desviarse, no ha aparecido en ningún intervalo de observación.

- Nivel 8: No necesita ninguna ayuda durante la totalidad de su desplazamiento pero en una ocasión, incluido el recorrido de comprobación, se ha desviado o perdido.

- Nivel 7: Necesita, como mucho, una o dos ayudas tipo 1. Realiza el recorrido total con un desvío o pérdida como máximo.

- Nivel 6: Necesita, como mucho, una ayuda tipo 2. Realiza el recorrido total con un solo desajuste en la movilidad. 
- Nivel S: Necesita más de dos ayudas 2. El recorrido lo realiza de forma idéntica a la descrita en las anteriores categorías.

- Nivel 4: Necesita, como mucho una, ayuda 3. Se le permite una frecuencia de dos desvíos o pérdidas en la suma total de intervalos de observación del recorrido final.

- Nivel 3: Necesita dos ayudas 3. Se permite una frecuencia de tres desajuste en la movilidad total.

- Nivel 2: Necesita dos ayudas 3. Además no es capaz de llegar a uno de los puntos de referencia durante el recorrido de comprobación.

- Nivel 1: Necesita una ayuda 4. No es capaz tampoco de llegar a uno de los mojones del recorrido de comprobación.

- Nivel 0: Necesita una ayuda 5, o bien no llega a ninguno de los dos puntos del recorrido de comprobación.

Para externalizar las representaciones de los sujetos se han utilizado dos tipos de métodos: uno cartográfico, consistente en la construcción de una maqueta del recorrido, y otro verbal, en el que debían estimar las distancias entre los 7 elementos de la ruta, siguiendo el método de comparaciones triádicas usado con invidentes por Rieser, Lockman y Pick (1981).

Las maquetas, se han analizado siguiendo los métodos tradicionales en los estudios sobre el desarrollo de la representación espacial: categorización de acuerdo con las etapas establecidas por Hart y Moore (1973) en sistemas de referencia «egocéntrico", «fijo» y «coordinado»; y análisis de la organización espacial de la maqueta mediante un análisis de conglomerados. En los dos casos las categorizaciones fueron hechas por tres jueces de forma independiente, siendo la correlación interjueces de 0.80 .

Las estimaciones de distancias se han sometido a un análisis multidimensional (programa TRISOSCAL de la Universidad de Edimburgo). Este análisis nos proporciona la disposición, en un eje euclidiano, de dos dimensiones de cada uno de los 7 lugares cuya distancia se ha estimado, de tal manera que podemos obtener una representación gráfica del espacio representado, similar a la que se obtenía en la maqueta. Así, posteriormente. se sometía esta respresentación gráfica a los mismos análisis que la maqueta: sistema de referencia y análisis de conglomerados.

\section{RESULTADOS}

La compleja estructura que tienen nuestros datos hace que sólo ofrezcamos aquí un resumen de sus aspectos más importantes. Remitimos al lector interesado al trabajo de Huertas (1989). En primer lugar exponemos los resultados obtenidos en el espacio pequeño, luego los relativos al espacio grande, posteriormente los datos de la comparación entre los dos tipos de espacio y, por último, los datos comparativos entre las diferentes técnicas con que se ha evaluado la movilidad y la representación espacial.

\subsection{Espacio pequeño}

\section{Medidas de representación}

El efecto del aprendizaje (es decir la diferencia entre los resultados obtenidos entre la primera sesión y la última), ha resultado igualmente signi- 
espacio $\mathrm{y}$, por último, los datos comparativos entre las diferentes técnicas con que se ha evaluado la movilidad y la representación espacial.

\subsection{Espacio pequeño}

\section{Medidas de reprèsentación}

El efecto del aprendizaje (es decir la diferencia entre los resultados obtenidos entre la primera sesión y la última), ha resultado igualmente signi-

\section{FIGURA 2}

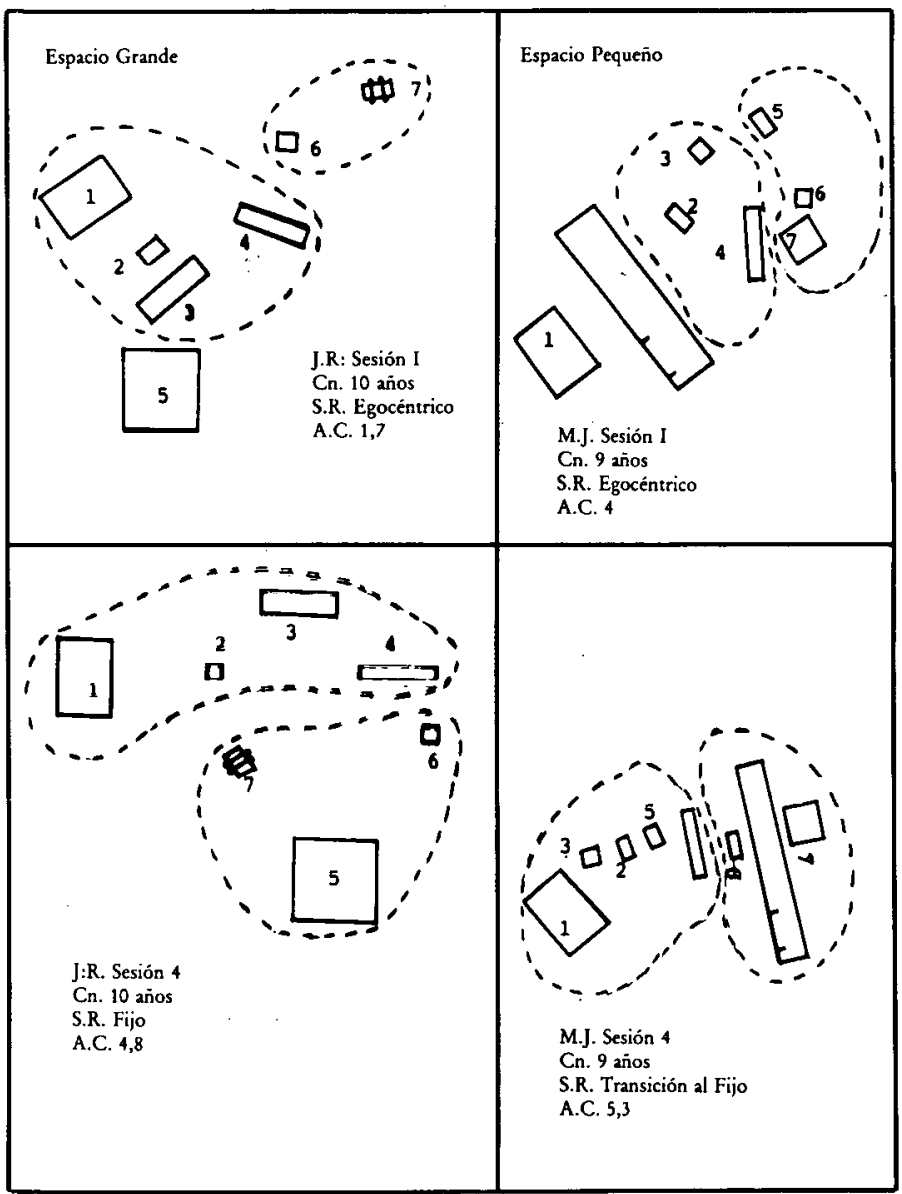

Ejemplos de la representación del entorno pequeño y grande, con las maquetas de dos niños, en la primera y ültima sesión de aprendizaje

ficativo (Wilcoxon, $\mathrm{p}<0.05$ ), en las dos medidas de representación utilizadas: maquetas y estimaciones de distancias (Tabla I). En ambas, el nivel medio de ganancia ha sido de un subestadio, tanto si se analizan los datos de los sujetos en conjunto, como si se consideran los distintos niveles de edad (Figura 1). En consecuencia, el tipo de aprendizaje a que se ha some- 
tido a los sujetos no consigue igualar las representaciones de los niños y las de los adolescentes. Tampoco ha sido diferente el aprendizaje entre el grupo de ciegos tardíos y precoces.

Tanto en las maquetas como en las estimaciones de distancias, se han encontrado diferencias significativas (Kolmogorov-Smirnov, $p<0.05$ ) respecto a la variable edad. Estas se centran en la comparación de los sujetos más pequeños (niveles I y II), con los mayores (niveles III y IV), igual para la primera sesión de aprendizaje que para la última, e independientemente de la experiencia visual (Tabla II).

Para ninguna de las dos medidas de representación, se han encontrado diferencias significativas en la variable experiencia visual, en cualquiera de los análisis realizados.

\section{Medidas de movilidad}

El efecto del aprendizaje no se refleja de forma homogénea en todas las medidas de movilidad utilizadas. Por lo que respecta al número y tipo de ayudas, al nivel de ajuste y a la duración del recorrido los análisis efectuados no muestran diferencias significativas entre la primera sesión y la última. Por el contrario, si consideramos el éxito alcanzado en la última sesión, se produce un aprendizaje aceptable en todos los sujetos que consiguieron un nivel superior a la media de éxito estipulada. Podemos considerar, por tanto, que es esta última medida la más sensible al efecto de aprendizaje.

También el efecto de la edad se muestra de forma distinta de acuerdo con la medida que se utilice. El número de ayudas requeridas es mayor en los sujetos pequeños que en los mayores; este efecto resulta claramente significativo (Kolmogorov-Smirnov, $\mathrm{p}<0.05$ ) cuando se comparan los niveles I y IV, en cualquiera de los cuatro ensayos. En el nivel de ajuste y el éxito alcanzado en la última sesión, las diferencias no alcanzan suficiente significación estadística, aunque hay una tendencia a que los sujetos mayores obtengan mejores puntuaciones que los pequeños. Por último $\mathrm{la} d u$ ración de recorrido no se muestra sensible a las diferencias evolutivas ( $\mathrm{Ta}$ bla III). Tampoco en estas medidas de movilidad se han encontrado diferencias que puedan considerarse significativas al comparar ciegos de nacimiento y ciegos tardíos, es decir, en relación con la variable "experiencia visualw.

\subsection{Espacio grande}

\section{Medidas de representación}

El efecto del aprendizaje es significativo tanto en las maquetas como en las estimaciones de distancias (aunque con esta última medida se muestra más claramente). La media de ganancia entre la sesión inicial y la última es de un subestadio, tanto para ciegos precoces como tardíos (Figura 2). Si analizamos el aprendizaje por niveles de edad, en todos se muestra significativo (Wilcoxon, $p<0.05$ ), excepto en el grupo de niños más pequeños.

La influencia de la edad se manifiesta como relevante en todas las medidas utilizadas (Tabla IV). En los dos tipos de análisis efectuados sobre las 
TABLA I

Puntuaciones medias en la representación, según la edad, tipo de prueba realizada, análisis de la misma y aprendizaje en el espacio pequeño

\begin{tabular}{|c|c|c|c|c|c|c|c|c|}
\hline \multirow{3}{*}{ Edad } & \multicolumn{4}{|c|}{ Maquetas } & \multicolumn{4}{|c|}{ Estimación de distancias } \\
\hline & \multicolumn{2}{|c|}{ Sist. de referencia } & \multicolumn{2}{|c|}{ Anal. conglomerados } & \multicolumn{2}{|c|}{ Sist. de referencia } & \multicolumn{2}{|c|}{ Anal. conglomerados } \\
\hline & S1 & $\$ 4$ & $\mathbf{S 1}$ & S4 & S1 & S4 & S1 & S4 \\
\hline I & 1.2 & 2.5 & 3.4 & 7.5 & 1.7 & 2.3 & 6.1 & 9.5 \\
\hline II & 1.5 & 2.5 & 8.3 & 11.2 & 2.2 & 3.2 & 5.1 & 7.1 \\
\hline III & 3.5 & 4.2 & 12.9 & 18.2 & 3.2 & 4.5 & 12.4 & 17.9 \\
\hline IV & 4.2 & 5.0 & 18.0 & 24.1 & 3.5 & 4.8 & 19.8 & 22.5 \\
\hline
\end{tabular}

S1 y $\$ 4=$ Sesión primera y última.

\section{TABLA II}

Medias de puntuaciones en la representación, según la edad y experiencia visual en el espacio pequeño

\begin{tabular}{|c|c|c|c|c|c|c|c|c|c|c|c|c|c|c|c|c|}
\hline \multirow{4}{*}{ Edad } & \multicolumn{8}{|c|}{ Maquetas } & \multicolumn{8}{|c|}{ Estimación de distancias } \\
\hline & \multicolumn{4}{|c|}{ Sist. de referencia } & \multicolumn{4}{|c|}{ Anal. conglomerados } & \multicolumn{4}{|c|}{ Sist. de referencia } & \multicolumn{4}{|c|}{ Anal. conglomerados } \\
\hline & \multicolumn{2}{|c|}{$\mathrm{CN}$} & \multicolumn{2}{|c|}{ CT } & \multicolumn{2}{|c|}{ CN } & \multicolumn{2}{|c|}{ CT } & \multicolumn{2}{|c|}{$\mathrm{CN}$} & \multicolumn{2}{|c|}{ CT } & \multicolumn{2}{|c|}{$\mathrm{CN}$} & \multicolumn{2}{|c|}{ CT } \\
\hline & S1 & S4 & S1 & $\$ 4$ & $\$ 1$ & $\$ 4$ & S1 & S4 & $\$ 1$ & S4 & S1 & $\$ 4$ & S1 & S4 & $\$ 1$ & S4 \\
\hline $1(\mathrm{I}+\mathrm{II})$ & 1.02 & 2.25 & 1.75 & 2.75 & 5.50 & 8.72 & 5.68 & 10.0 & 1.50 & 2.25 & 1.50 & 2.25 & 7.31 & 12.25 & 3.96 & 4.37 \\
\hline $2(\mathrm{III}+\mathrm{IV})$ & 4.00 & 4.50 & 3.50 & 4.50 & 17.122 & 22.151 & 10.502 & 20.25 & 3.75 & 4.50 & 2.50 & 4.75 & 17.60 & 20.37 & 14.62 & 20.12 \\
\hline
\end{tabular}

CN: Ciegos de nacimiento; CT: Ciegos tardios.

\section{TABLA III}

Puntuaciones medias en las medidas de movilidad, según la edad y la sesión de entrenamiento en el espacio pequeño

\begin{tabular}{|c|c|c|c|c|c|c|c|}
\hline \multirow{2}{*}{ Edad } & \multicolumn{2}{|c|}{ Número de ayudas } & \multicolumn{2}{|c|}{ Ajuste en mov. } & \multicolumn{2}{|c|}{ Duración del mov. } & \multirow{2}{*}{$\frac{\text { Nivel de éxito }}{\text { S4 }}$} \\
\hline & S1 & S4 & S1 & S4 & S1 & S4 & \\
\hline $\begin{array}{l}\text { I } \\
\text { II } \\
\text { III } \\
\text { IV }\end{array}$ & $\begin{array}{l}7.5 \\
6.5 \\
2.0 \\
1.0\end{array}$ & $\begin{array}{l}3.5 \\
5.5 \\
0.25 \\
2.0\end{array}$ & $\begin{array}{l}0.4 \\
0.4 \\
0.6 \\
0.6\end{array}$ & $\begin{array}{l}0.6 \\
0.6 \\
0.7 \\
0.4\end{array}$ & $\begin{array}{r}13.2 \\
16.5 \\
9.7 \\
11.2\end{array}$ & $\begin{array}{r}12.0 \\
15.5 \\
8.0 \\
15.0\end{array}$ & $\begin{array}{r}4.5 \\
4.75 \\
7.25 \\
6.0\end{array}$ \\
\hline
\end{tabular}

S1 y $\mathbf{S 4}=$ Sesión primera y última.

maquetas se han encontrado diferencias claramente significativas (Kolmogorov-Smirnov, $\mathrm{p}<0.05$ ) cuando se comparan los niveles de edad I y II con el III y el IV (Tabla V). No obstante, en las estimaciones de distancias el efecto de esta variable es menor y sólo adquiere niveles de significación adecuados cuando se comparan los tres primeros niveles de edad respecto al cuarto. 


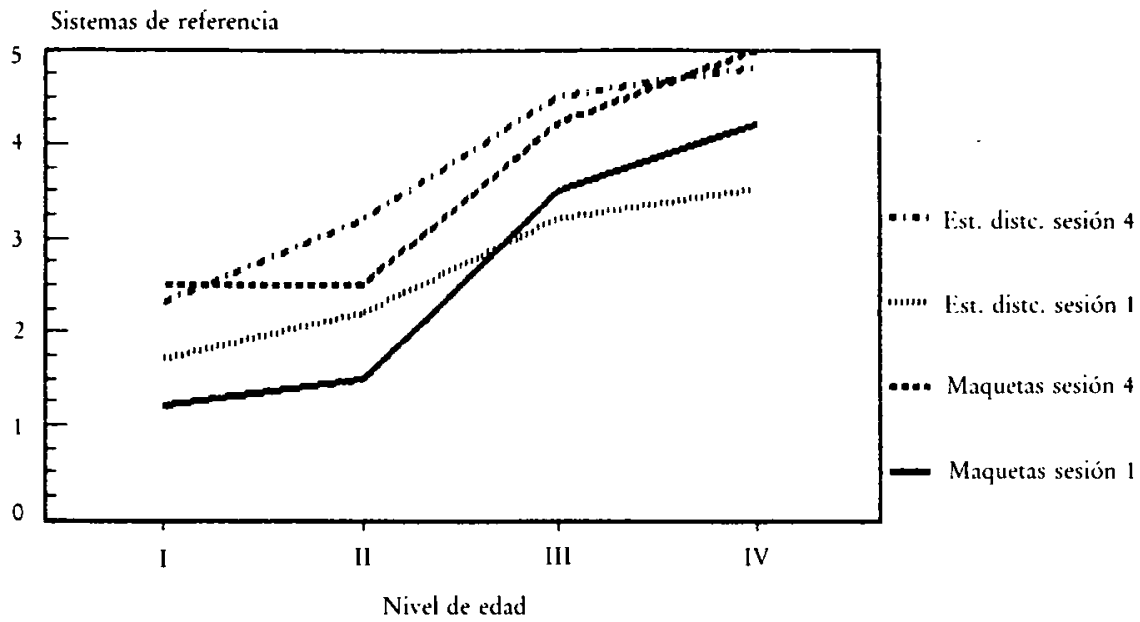

Espacio pequeño. Puntuaciones en los sistemas de referencia en maquetas y estimacion de distancias (MDS)

Tampoco en este caso la experiencia visual parece incidir en la representación de los sujetos ciegos, ya que no se han encontrado diferencias significativas entre ciegos tempranos y tardíos en ninguna de las medidas. No obstante, existe una tendencia a que los ciegos tardíos realicen representaciones más adecuadas que los tempranos, sobre todo en el grupo de menor edad.

\section{Medidas de movilidad}

El efecto del aprendizaje se manifiesta de forma desigual en las distintas medidas. En concreto, el numero global de ayudas que necesitaron los sujetos fue menor en la última sesión. El ajuste, o marcha adecuada aumentó, si bien las diferencias no son significativas estadísticamente. El nivel de éxito alcanzado en la última sesión aparece modulado por la variable edad, ya que sólo los sujetos de los últimos niveles de edad (13-18), alcanzaron el nivel de éxito suficiente que significa haber aprendido el recorrido. De nuevo la medida del tiempo no ha arrojado resultados significativos (Tabla VI).

La variable edad ha tenido efectos significativos (Kolmogorov-Smirnov, $p<0.05)$ en tres de los procedimientos utilizados: número de ayudas, nivel de ajuste y éxito. Este efecto se manifiesta claramente al comparar los dos primeros niveles de edad con los dos últimos. La duración del recorrido no mostró diferencias respecto a la edad.

Tampoco en este caso ha habido diferencias relacionadas con la experiencia visual.

\subsection{Comparación entre los dos tipos de espacios}

No se han encontrado diferencias con significación estadística al comparar los dos espacios en ninguna de las técnicas utilizadas para objetivar las representaciones de los ciegos, tanto cuando las comparaciones se hacen 
TABLA IV

Puntuaciones medias en la representación, según la edad, tipo de prueba realizada, análisis de la misma y aprendizaje en el espacio grande

\begin{tabular}{|c|c|c|c|c|c|c|c|c|}
\hline \multirow{3}{*}{ Edad } & \multicolumn{4}{|c|}{ Maquetas } & \multicolumn{4}{|c|}{ Estimación de distancias } \\
\hline & \multicolumn{2}{|c|}{ Sist. de referencia } & \multicolumn{2}{|c|}{ Anal. conglomerados } & \multicolumn{2}{|c|}{ Sist. de referencia } & \multicolumn{2}{|c|}{ Anal conglomerados } \\
\hline & S1 & S4 & S1 & $\$ 4$ & $\mathbf{S 1}$ & S4 & S1 & S4 \\
\hline I & 1.5 & 1.9 & 4.3 & 5.0 & 1.5 & 2.1 & 3.0 & 7.1 \\
\hline II & 1.1 & 2.3 & 3.3 & 4.3 & 1.7 & 2.2 & 4.5 & 7.3 \\
\hline III & 3.3 & 3.7 & 12.0 & 12.2 & 2.5 & 3.4 & 9.0 & 12.3 \\
\hline IV & 3.6 & 4.8 & 15.0 & 16.9 & 3.6 & 4.7 & 13.1 & 20.9 \\
\hline
\end{tabular}

S1 y S4 = Sesión primera y última.

\section{TABLA V}

Medias puntuaciones en la representación, según la edad y la experiencia visual en el espacio grande

\begin{tabular}{|c|c|c|c|c|c|c|c|c|c|c|c|c|c|c|c|c|}
\hline \multirow{4}{*}{ Edad } & \multicolumn{8}{|c|}{ Maquetas } & \multicolumn{8}{|c|}{ Estimaciones de distancias } \\
\hline & \multicolumn{4}{|c|}{ Sist. de referencia } & \multicolumn{4}{|c|}{ Anal. conglomerados } & \multicolumn{4}{|c|}{ Sist. de referencia } & \multicolumn{4}{|c|}{ Anal. conglomerados } \\
\hline & \multicolumn{2}{|c|}{$\mathrm{CN}$} & \multicolumn{2}{|c|}{ CT } & \multicolumn{2}{|c|}{$\mathrm{CN}$. } & \multicolumn{2}{|c|}{ CT } & \multicolumn{2}{|c|}{$\mathrm{CN}$} & \multicolumn{2}{|c|}{ CT } & \multicolumn{2}{|c|}{$\mathrm{CN}$} & \multicolumn{2}{|c|}{$\mathrm{CT}$} \\
\hline & S1 & SA & S1 & S4 & S1 & S4 & S1 & S4 & S1 & S4 & S1 & S4 & S1 & S4 & S1 & S4 \\
\hline & & & & & & & & & & & & & & & & \\
\hline $\begin{array}{l}1(\mathrm{I}+\mathrm{II}) \\
2(\mathrm{III}+\mathrm{IV})\end{array}$ & $\begin{array}{l}1.00 \\
3.12\end{array}$ & $\begin{array}{l}2.00 \\
4.07\end{array}$ & $\begin{array}{l}1.65 \\
3.80\end{array}$ & $\begin{array}{l}2.25 \\
4.47\end{array}$ & $\begin{array}{r}2.81 \\
11.60\end{array}$ & $\begin{array}{r}4.17 \\
16.17\end{array}$ & $\begin{array}{r}4.75 \\
15.42\end{array}$ & $\begin{array}{r}4.87 \\
16.12\end{array}$ & $\begin{array}{l}1.50 \\
2.80\end{array}$ & $\begin{array}{l}2.15 \\
4.15\end{array}$ & $\begin{array}{l}1.75 \\
3.37\end{array}$ & $\begin{array}{l}2.15 \\
3.97\end{array}$ & $\begin{array}{r}3.50 \\
10.87\end{array}$ & $\begin{array}{r}6.57 \\
18.82\end{array}$ & $\begin{array}{r}4.02 \\
12.87\end{array}$ & $\begin{array}{r}7.82 \\
14.40\end{array}$ \\
\hline
\end{tabular}

$\mathrm{CN}$ : Ciegos de nacimiento; $\mathrm{CT}$ : Ciegos tardíos.

\section{TABLA VI}

Puntuaciones medias en las medidas de movilidad, según la edad y la sesión de entrenamiento en el espacio grande

\begin{tabular}{|c|c|c|c|c|c|c|c|}
\hline \multirow{2}{*}{ Edad } & \multicolumn{2}{|c|}{ Número de ayudas } & \multicolumn{2}{|c|}{ Ajuste en mov. } & \multicolumn{2}{|c|}{ Duración del mov. } & \multirow{2}{*}{$\frac{\text { Nivel de éxito }}{\text { S4 }}$} \\
\hline & S1 & S4 & S1 & S4 & S1 & S4 & \\
\hline $\begin{array}{l}\text { I } \\
\text { II } \\
\text { III } \\
\text { IV }\end{array}$ & $\begin{array}{r}11.7 \\
8.7 \\
2.0 \\
1.2\end{array}$ & $\begin{array}{l}9.0 \\
6.2 \\
1.7 \\
1.0\end{array}$ & $\begin{array}{l}0.5 \\
0.5 \\
0.7 \\
0.8\end{array}$ & $\begin{array}{l}0.4 \\
0.6 \\
0.8 \\
0.8\end{array}$ & $\begin{array}{l}22.5 \\
17.7 \\
19.7 \\
17.2\end{array}$ & $\begin{array}{l}22.0 \\
17.0 \\
18.0 \\
16.0\end{array}$ & $\begin{array}{l}1.7 \\
3.0 \\
6.5 \\
7.2\end{array}$ \\
\hline
\end{tabular}

\$1 y $\$ 4=$ Sesión primera y última.

de forma global como cuando se agrupan de acuerdo con las distintas variables. No obstante, existe cierta tendencia en los datos a que las representaciones del espacio pequeño sean algo mejores que las del grande. En cualquier caso, la variable tamaño del espacio, tal y como ha sido manipulada en nuestro diseño, no determina la capacidad de representación en las personas ciegas. 
Anälisis de conglomerados.

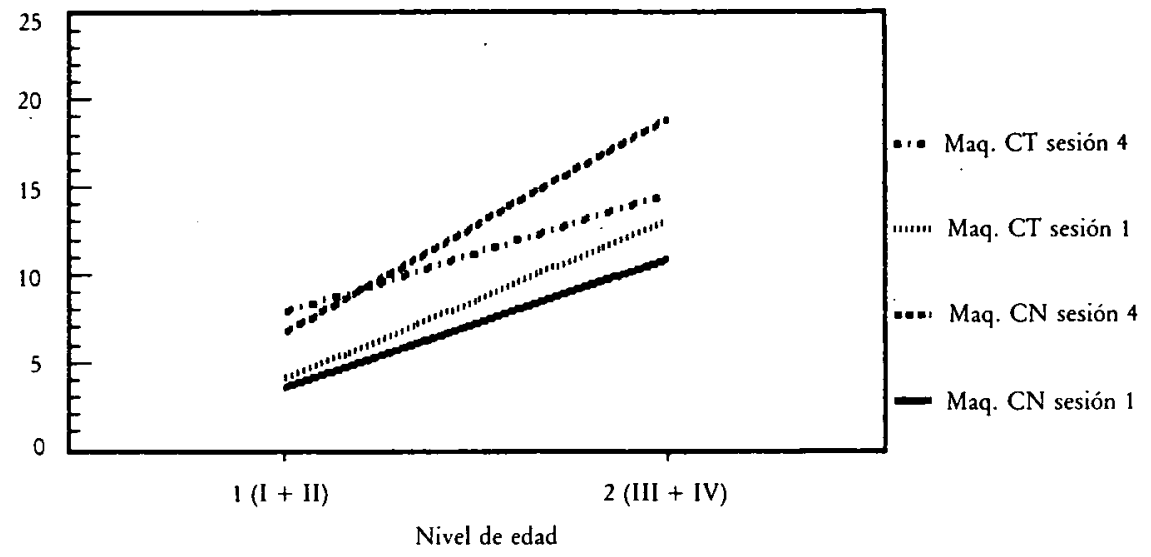

Espacio grande. Puntuaciones en análisis de conglomerado según la experiencia visual

Tampoco en las medidas de movilidad o conducta espacial se han encontrado diferencias claras entre uno y otro espacio. Globalmente, el número de ayudas que necesitaron los sujetos ha sido independiente del tamaño del espacio, aunque hay una cierta tendencia a que se necesite un número menor de ellas en el espacio pequeño sobre todo en los niños más pequeños y sobre todo en el caso de los ciegos sin experiencia visual. Respecto al ajuste de la marcha tampoco puede hablarse de diferencias significativas. En lo que se refiere al éxito alcanzado en la última sesión, hay ciertas diferencias en los dos primeros niveles de edad, los sujetos más jóvenes - terminaron conociendo mejor la ruta del espacio pequeño que la del grande.

\subsection{Comparación entre las distintas técnicas}

Al igual que en un experimento previo (Huertas y Ochaíta, 1988), la correlación media entre las dos técnicas de representación utilizadas - maquetas y estimaciones de distancias - ha sido alta y significativa (entre 0.785 y 0.976 ). En consecuencia, podemos afirmar que ambos procedimientos son igualmente útiles para exteriorizar las representaciones espaciales de los niños y adolescentes ciegos, aunque los más pequeños tengan ciertas dificultades en la estimación de distancias.

También las medidas relativas a la conducta espacial, tienen entre sí correlaciones altas y, en general, significativas (entre 0.60 y 0.85 ). Las correlaciones más altas son las que resultan de la comparación entre las ayudas y el éxito final. Por el contrario el tiempo no parece estar relacionado con las restantes medidas de movilidad.

Si comparamos las medidas de representación con las de movilidad obtenemos los siguientes resultados. Las correlaciones con las ayudas que recibían los sujetos oscilan entre 0.60 y 0.80 independientemente de la edad y del aprendizaje y del tamaño del espacio. La comparación con el éxito alcanzado en el último recorrido alcanza puntuaciones que van de 0.78 a 0.81 para el espacio grande y de 0.35 a 0.54 en el pequeño. Las correlacio- 
nes entre maquetas/estimaciones de distancias y el ajuste de la marcha oscilan entre 0.50 y 0.70 . Por último no hay relaciones respecto a la duración del recorrido.

\section{DISCUSION, CONCLUSIONES E IMPLICACIONES EDUCATIVAS}

En términos generales podemos concluir que las variables tamaño del espacio y experiencia visual no han resultado ser importantes en nuestra investigación. Son el desarrollo y el aprendizaje, los factores que inciden de forma clara en los resultados.

El tamaño del espacio, no ha resultado ser, con nuestro diseño, un factor que explique los resultados obtenidos. Pensamos, con Warren (1987), que es la complejidad y no el tamaño la variable que incide en la capacidad de los ciegos para conocer y representarse los distintos entornos, aunque en general complejidad y tamaño suelen presentarse unidas (tanto en los diseños experimentales como en los entornos naturales). No obstante existe en nuestros datos la tendencia a que los sujetos de menor edad conozcan y representen mejor la ruta correspondiente al espacio pequeño que la del grande. Es posible que esta tendencia aumentase si la diferencia de tamaño entre los dos espacios estudiados hubiera sido mayor. En consecuencia, se necesitan nuevas investigaciones sobre la incidencia del tamaño y, sobre todo, la complejidad del espacio, en la movilidad y representación de los invidentes de distintas edades.

Tampoco la experiencia visual previa a la ceguera, es un factor que facilite el conocimiento y representación de los espacios implicados en nuestro diseño. Nuestros datos concuerdan en esto con trabajos anteriores (Fletcher, 1981; Hudson, 1984): cuando el espacio que ha de aprenderse tiene una estructura de rutas y ésta no es muy complicada, no existen diferencias entre ciegos tempranos y ciegos tardíos.

El nivel de desarrollo, determinado en este trabajo por la variable edad, es un factor fundamental en la capacidad de los ciegos para representarse un determinado espacio, ya que, independientemente del tamaño del mismo, existe una notable diferencia entre los niños pequeños (niveles I y II), y los mayores (niveles III y IV). Por tanto, a partir de los 13 años aumenta notablemente la capacidad para representarse un espacio, capacidad que se hace más marcada a los 17 , aunque no se estabiliza a esta edad, sino que sigue progresando hasta aproximadamente los 18 años. Estos datos concuerdan con los obtenidos por nuestro equipo de investigación, tanto para el desarrollo cognitivo (Rosa y col., 1986; Ochaíta y col., 1988) como en la adquisición de la lectura braille (Ochaíta y col., 1988), y pueden ser explicados en términos de acceso a un pensamiento de tipo verbal - proposicional que permite al adolescente ciego «re-mediar», en cierta medida, su déficit en los aspectos figurativos.

Las pautas o etapas de desarrollo de la representación espacial en los ciegos son. semejantes a las obtenidas en videntes por Hart (1979), Martín (1985) y Aragonés (1988), aunque con un retraso de 2 ó 3 años respecto a éstos, retraso que empieza a superarse, precisamente, sobre los 13 años.

La experiencia con el entorno, o aprendizaje, incide en la capacidad de 
los invidentes para representarse el entorno, aunque, en nuestro diseño el avance microgenético se ve limitado al avance ontogenético. En todos los niveles de edad la ganancia obtenida entre las representaciones primera y última ha sido, en términos generales, de un subestadio, sin que la experiencia con el entorno haya disminuido las diferencias entre los distintos niveles de edad. No obstante, nuestras conclusiones en este sentido no pretenden ser definitivas: es posible que con más sesiones u otro procedimiento de instrucción, se hubiera conseguido un mayor avance microgenético incluso en los niños más pequeños.

Por lo que respecta a las medidas de movilidad y conocimiento de la ruta, desarrollo y aprendizaje comparten la influencia en los resultados. El número de ayudas solicitadas disminuye en todas las edades, gracias a la experiencia con el entorno. La medida de éxito en la última sesión pone de manifiesto que todos los sujetos adquieren, gracias al aprendizaje un conocimiento aceptable del espacio pequeño. Sin embargo, cuando el entorno es más grande sólo los sujetos de mayor edad tienen un éxito medio en el recorrido. El ajuste en la movilidad, parece ser una habilidad ya adquirida incluso para nuestros sujetos más jóvenes, por lo que no se ve afectada ni por el desarrollo ni por la experiencia. Por último el tiempo de duración del recorrido, no se ha revelado como una medida adecuada.

Tanto las maquetas como las estimaciones de distancias se revelan como técnicas adecuadas para exteriorizar las representaciones espaciales de los ciegos. Las altas correlaciones obtenidas entre ellas, pueden deberse a que han sido analizadas de la misma manera, pero no puede desecharse la hipótesis de que ambos procedimientos puedan ser resueltos por los ciegos mediante un pensamiento proposicional. Los resultadosaconsejan seguir investigando sobre las posibilidades del análisis multidimensional de las estimaciones verbales de distancias como método para evaluar las representaciones del entorno en los ciegos.

La correlación entre las medidas de movilidad y representación es, como se esperaba, muy alta en las personas ciegas. En ausencia de la anticipación perceptiva que proporciona la visión, los invidentes tienen que acudir a sus representaciones de un entorno (anticipación cognitiva), para poder moverse por el mismo con cierta soltura.

Por último, es necesario apuntar algunas de las repercusiones que este trabajo tiene para la instrucción en movilidad y conocimiento espacial de los niños y adolescentes ciegos. En primer lugar se hace necesario que las técnicas de instrucción estén basadas en un sustrato teórico, como el que sirve de marco a este trabajo. Por otro lado, es importante que los profesionales que trabajan con los invidentes conozcan las técnicas más adecuadas para evaluar el éxito en las técnicas de instrucción y movilidad. Por último señalar que mediante el aprendizaje (incluso sin una instrucción explícita como la del presente trabajo), es posible incrementar no sólo la movilidad sino también la representación de los invidentes, aunque consideramos imprescindible adaptar las técnicas instruccionales al nivel de desarrollo de los niños. 


\section{Referencias}

APPLEYARD, D. D. (1969). «Why buildings a predictive tool for architects and planners». Environment and behavoir, vol. 1, pp. 131-156.

- (1970). "Styles and Methods of Structuring a City". Environment and Bebavior, vol. 2, pp. 100-117.

Aragonés, J. I.; Jiménez, C.; MATIAs, C., y Noguerol, V. (1988). Experiencias del niño en su entorno urbano. Madrid, M.O.P.U.

BECK, R. J., y WOOD, D. (1976). "Cognitive Transformation of information from urban geographie fields to mental mapsw. Environment and Behavior, vol. 8 (2), pp. 199-238.

De VegA, M. (1984). Introducción a la Psicología Cognitiva. Madrid, Alianza.

DOwNS, R. M., y STEA, D. (1973). «Cognitive maps and spatial behavior: Process and productsm. En R. M. Downs y D. Stea: Image and Environment. Chicago, Aldine.

Evans, G. W. (1980). «Environmental cognition». Psychologycal Bulletin, vol. 88 (2), pp. 259-287.

FLETCHER, J. F. (1981). «Spatial representation in blind children: 2. Effect of taks variations». Journal of Visual Impairment and Blindness, 75, pp. 46-49.

FOULKE, E. (1982). «Percep̃tion, cognition and mobility of blind pedestrian». En Potegal (ed.): Spatial Abilities. Nueva York. Academic Press.

HART, R., y MOORE, G. T. (1973). «The development of spatial cognition: A review". En R. Downs y D. Stea (eds.): Image and environment. Cognitive mappeing and spatial bebavior. Chicago. Aldine Publishing Co.

HART, R. (1979). Children's experience of place. New York. Irvington.

HOLLYFIELD, R. L. y FOULKE, E. (1983). "The spatial cognition and blind pedestrians». Journal of visual impairment and blidness.

HuDSON, L. M. (1984). «Measurement of mental representation of space in visual handicapped». Comunicación presentada en Lousville Space Conference. Lousville, Kentucky.

HuERTAS, J. A., y OchaitA, E. (1988). „Diferentes procedimientos de externalización de la representación espacial. Un estudio evolutivo con niños ciegos». Estudios de Psicología, 36, pp. 53-74.

MARCHESI, A. (1983). «Conceptos espaciales, mapas cognitivos y orientación en el espacio». Estudios de Psicologia, 14/15, pp. 85-92.

MARTÍN, E. (1985). La representación espacial del entorno en los niños. Una comparación entre el medio urbano y el medio rural. Tesis Doctoral no publicada. Universidad Complutense.

MOORE, G. T. (1973). «Developmental differences in environmental cognition». En W. Preiser (ed.): Environmental design Research. Dowden, Hutchinson y Roos. Stroudsburg.

- (1974). "The development of environmental knowing: An overview of an interaccional -constructi- vist theory and some data within individual development variations. En D. Canter y T. Lee (eds.): Psychology and the built environment. Architectural Press Ltd. Tonbridge.

OCHAÍTA, E., y HuerTAS, J. A. (1988). "Conocimiento del espacio, representación y movilidad en las personas ciegas». Infancia y Aprendizaje, 43.

Piaget, J., e InHELDer, B. (1947). La géometre spontanée de l'enfant. París, P.U.F.

RIESER, J. J.; LOCKMAN. J. J., y PICK, H. L. (1981). "Assesing blind travelers knowledge of spatial layout". Journal of Visual Impairments and Blindness, pp. 321-326.

Stokols, D., y ALtman, I. (1987). Handbook of environmental Psychology. A Wiley-Interscience publication.

VigotSKY, L. S. (1934). Pensamiento y Lenguaje. Buenos Aires. La Pléyade.

WARREN, D. H. (1987). "Blindness and early childhood development». Comunicación presentada al Second International Symposium on Prevention and Intervention in Childbood and Youth. Conceptual and Metodological Issues. Bielefled, Alemania Federal.

\section{Extended summary}

The aim of this research is the joint study of the main factors - relative both to the subject and space- which may determine environmental knowledge and representation in blind people: age, previous visual experience, degree of familiarity with space (learning) and size of that space, especifically emphasizing relations between ontogenesis and microgenesis. A previous review on the litterature (Ochaita and Huertas, 1989), highlights that 
the absence of definitive conclusion may be due to the lack of joint studies on the incidence of all these factors on the capacity of blind subjects to be acquainted with the space surrounding them. In addition, research addressing this issue from a developmental viewpoint is practically non-existent; there is not one in which development and learning are related, in spite of its importance for orientation and movility instruction to blind adolescents.

A mixed theoretical framework was adopted, taken from environmental psychology (Evans, 1980; Lynch, 1960), developmental psychology of spatial knowledge (Piaget and Inhelder, 1947; Piaget, Inhelder and Szeminska, 1948; Hart, 1979; Moore, 1974; Siegel, 1975), and cognitive psychology (De Vega, 1984).

The study sample were two groups of blind children (congenital and adventitiously blind), divided into four age levels with a mean age of 9,11 , 14 , and 17 years. The procedure consisted in two independent samples learning an unknown environment (large/small) in a maximum of four trials and receiving different types of help. The route involved in learning each of the two environments was made up of seven elements. Subject's knowledge and spatial representation was evaluated through three different techniques of external representation, which a previous study (Huertas and Ochaita, 1988) had found adequate for using with blind children and adolescents. In particular, a cartographic technique was used -construction of a scale model - together with a verbal procedure involving distance estimates, and a behavioural technique or observation of the subjects' actual movements.

Three different judges analized the scale models following the methodology and stages proposed by Hart and Moore (1973): systems of reference and cluster analysis. Distance estimates were also carried out following the procedure described by Lockman, Rieser and Pick (1981). Subjects made judgements of the seven elements in the environment, taken three at a time, estimating their distance in terms of which ones were place at a longer, shorter or intermediate distance but without following a metric system. Multidimensional scaling was applied to the data with the use of the «trisoscal» method from the MDS (X) package from Edinburg University. Finally, subject's learning routes were analysed thourgh observational procedures.

For each environments, first, data from scale models were analysed, then data obtained through distance estimates, and lastly the results from behavioural maps. For each of these procedures of external representation the following was studied: effect of development; learning capacity, evaluated as the improvement observed between the first and final trial; and the effect of visual experience. The results obtained through the different procedures were then compared, and finally the data from the two different environments was also compared.

Results show that subjects' age is the one factor which has greater incidence on the spatial knowledge and representation of blind children and adolescents. Learning is an important factor but, at least in the present design, it appears to be subordinated to development, for the average improvement observed between the first and final trial signified the progression from one stage to the next. Results from the group with a visual experience prior to their blindness were not significantly different, however there 
is a certain tendencency for adventitiously blind subjects to carry out more precise representations of the environment. Size of space is not, in this case, a determining factor of knowledge of the environment. Finally, the present results support previous findings (Huertas and Ochaita, 1988) on the validity of the methods used to evaluate spatial knowledge in blind children and adolescents. Here the usefulness of multidimensional scaling in evaluating spatial representation in blind people is underlined.

These results, on the capacity of blind children and adolescents to represent the environment in the absence of a sensory input of such importance as vision, are of unquestionable theoretical interest to cognitive and developmental psychology. From an educational viewpoint, this study has clear implications for orientation instruction, mobility, and knowledge of the environment in blind schoolchildren. 